\title{
A GENERALIZATION OF THE COHOMOLOGY OF GROUPS
}

\author{
STEFAN WANER
}

\begin{abstract}
Generalizations of the cohomology of finite groups, in which one considers varying families of subgroups, are presented. These groups are shown to relate to Bredon equivariant of homology of universal $G$-spaces, and to lead to necessary algebraic conditions for $G$-actions on contractible spaces.
\end{abstract}

1. Introduction. In this note, we present new algebraic invariants of a finite group $G$ generalizing the notion of cohomology of $G$. Specifically, given a collection $\mp$ of subgroups of $G$ closed under conjugation, as well as cohomological ("Hecke") functors $T$ and $T^{\prime}$ in the sense of $[\mathbf{G 1}]$, we construct groups $H^{*}\left((T, \mathcal{F}) ; T^{\prime}\right)$ with the property that $H^{*}((\hat{\mathbf{Z}},\{1\}) ; \hat{A}) \cong H^{*}(G ; A)$ for any $\mathbf{Z} G$-module $A$, where, for any $\mathbf{Z} G$-module $B, \hat{B}$ denotes the associated Hecke functor $\hat{B}(G / H)=B^{H}$. (The notion of a Hecke functor will be reviewed in §2.) The potential for such generalizations is alluded to in [RS].

As a topological application, we show in $\S 3$ that $H^{*}\left((T, \mathcal{F}) ; T^{\prime}\right)$ represents the equivariant Bredon cohomology of an associated universal $G$-space when $T=\mathbf{Z}$. (The constructions in $\S 2$ therefore give one an explicit computational machine for these cohomology groups.) In addition, we show that if $G$ acts on a suitable finite contractible complex with specified orbit types, then $H^{*}\left((T, \mathcal{F}) ; T^{\prime}\right)$ must vanish above the dimension of the complex.

The author is grateful to Professor Leonard Scott for his suggestions, particularly for his novel definition of a Hecke functor.

2. Mackey functors and the Bar resolution. First, we set up a few categories. Denote by $\mathcal{G}$ the category whose objects are the $G$-sets $G / H$ with $H \subset G$ and whose morphisms are the equivariant maps. Thus a morphism $f: G / K \rightarrow G / H$ must have the form $f(g K)=g g^{\prime} H$, where $K \subset g^{\prime} H^{\prime-1}$. Z $\mathcal{G}$ will denote the category whose objects are those of $\mathcal{G}$ but whose morphisms $G / K \rightarrow G / H$ are the $\mathbf{Z} G$-module homomorphisms $\mathbf{Z} G / K \rightarrow \mathbf{Z} G / H$, where $\mathbf{Z} G / J$ denotes the free $\mathbf{Z}$-module on the $G$-set $G / J$. If $₹$ is a family of subgroups of $G$ closed under conjugation, then we may define associated categories $\mathcal{G}(\mathcal{F})$ and $\mathbf{Z} \mathcal{G}(\mathcal{F})$ by considering, respectively, the full subcategories of $\mathcal{G}$ and $\mathbf{Z} \mathcal{G}$ whose objects are those $G / H$ with $H \in \mathcal{F}$.

Recall that a coefficient system in the sense of Bredon [B1] is a contravariant functor $T: \mathcal{G} \rightarrow A b$, where $A b$ is the category of abelian groups. A Hecke functor is usually thought of as a bifunctor $\left(T^{*}, T_{*}\right): \mathcal{G} \rightarrow A b$ where $T^{*}$ is contravariant and $T_{*}$ is covariant, obeying certain axioms (see [G1]). Following is what I believe to be the most succinct definition of a Hecke functor, suggested to me by Leonard Scott.

DEFINITION 2.1. A Hecke functor is an additive contravariant functor

$$
T: \mathbf{Z} \mathcal{G} \rightarrow A b .
$$

Received by the editors August 26, 1981 and, in revised form, December 10, 1981 . 1980 Mathematics Subject Classification. Primary 54H15.

(C) 1982 American Mathematical Society 0002-9939/81/0000-0725/\$02.75 
More generally, an $₹$-Hecke functor is an additive contravariant functor

$$
T: \mathbf{Z} \mathcal{G}(\mathcal{F}) \rightarrow A b .
$$

The collection of $\mathcal{F}$-Hecke functors forms a category whose morphisms are the natural transformations.

REMARKS 2.2. The natural inclusion $\mathcal{G} \rightarrow \mathrm{Z} \mathcal{G}$ displays every Hecke functor as, in particular, a coefficient system. Further, the inclusion $\mathbf{Z} N(H) \rightarrow \mathbf{Z G}(G / H, G / H)$ displays each $T(G / H)$ as a $\mathbf{Z} N(H)$-module, whose $N(H)$ denotes the normalizer of $H \subset G$. It is not hard to show that our definition of a Hecke functor is equivalent to Green's. It is, however, stronger than the notion of a Mackey functor as in, for example, [D1].

EXAMPLES OF HECKE FUNCTORS 2.3. If $R$ is a $\mathbf{Z} G$-module, one obtains an associated $\mathcal{F}$-Hecke functor, $\hat{R}$, as follows. If $H \in \mathcal{F}$, take $\hat{R}(G / H)=R^{H}$, and if $f: \mathbf{Z} G / K \rightarrow \mathbf{Z} G / H$ is specified by $f(e K)=\sum n_{i} g_{i} H$, one may take $\hat{R}(f): \hat{R}(G / H) \rightarrow \hat{R}(G / K)$ to be given by $\hat{R}(f)(a)=\sum n_{i} g_{i} a$. In particular, if $R$ is a trivial $\mathbf{Z} G$-module, one obtains the constant $₹$-Hecke functor $\hat{R}(G / H)=R$ for each $H \in \mathcal{F}$.

CONSTRUCTION 2.4. Fix an $\mathcal{F}$-Hecke functor $T$, and denote $\mathbf{Z} \mathcal{G}(\mathcal{F})$ by $C$ for brevity. Let $W_{*}(T, \mathcal{F})$ be the d.g. Z $G$-module given by

$$
W_{n}(T, \mathcal{F})=\bigoplus \mathrm{Z} G / H_{0} \otimes\left[C\left(G / H_{0}, G / H_{1}\right) \otimes \cdots \otimes C\left(G / H_{n-1}, G / H_{n}\right)\right] \otimes T\left(G / H_{n}\right),
$$

where the sum is taken over all distinct sequences $\left(H_{0}, \ldots, H_{n}\right)$ of subgroups in ₹. The action of $\mathbf{Z} G$ is the natural left action, and we define $\mathbf{Z} G$-homomorphisms $d_{n}: W_{n}(T, \mathcal{F}) \rightarrow W_{n-1}(T, \mathcal{F})$ by $d_{n}(x)=\sum_{i=0}^{n}(-1)^{i} F_{i}(x)$, where $F_{i}$ is given on generators by

$$
F_{i}\left(h\left[f_{1}, \ldots, f_{n}\right] t\right)= \begin{cases}f_{1}(y)\left[f_{2}, \ldots, f_{n}\right] t & \text { if } i=0 \\ y\left[f_{1}, \ldots, f_{n-1}\right] f_{n}^{*}(t) & \text { if } i=n \\ y\left[f_{1}, \ldots, f_{i} f_{i+1}, \ldots, f_{n}\right] t & \text { otherwise. }\end{cases}
$$

That $d_{*}$ is indeed a differential of this graded $\mathbf{Z} G$-module is easy to check.

Construction 2.4 may be thought of as a two-sided algebraic Bar construction $B_{*}(0, \mathbf{Z} \mathcal{G}(\mathcal{F}), T)$ analogous to Elmendorf's geometric construction in [E1]. Here, 0 denotes the object space of $\mathbf{Z} \mathcal{G}(\mathcal{F})$, and the general construction is fully explained in $[\mathbf{E 1}$.

We consider now the basic properties of $W_{*}(T, \mathcal{F})$.

Proposition 2.5. Let $H \in \mathcal{F}$. Then $\left(W_{*}(T, \mathcal{F})^{H}, d_{*}^{H}\right)$ is a resolution of $T(G / H)$ by $\mathbf{Z N}(H)$-modules.

ProOF. We have a natural isomorphism

$$
\sigma: W_{*}(T, \mathcal{F})^{H} \cong \operatorname{Hom}_{\mathbf{Z} G}\left(\mathbf{Z} G / H, W_{*}(T, \mathcal{F})\right)
$$

of $\mathbf{Z} N(H)$-modules, specified by sending $x$ to the unique $\mathbf{Z} G$-map $f: \mathbf{Z} G / H \rightarrow$ $W_{*}(T, \mathcal{F})$ specified by $f(e H)=x$.

Further,

$$
\begin{aligned}
\operatorname{Hom}_{\mathbf{Z} G}(\mathbf{Z} G / H, \mathbf{Z} G / K \otimes M) & \cong \operatorname{Hom}_{\mathbf{Z} G}(\mathbf{Z} G / H, \mathbf{Z} G / K) \otimes M \\
& =\mathbf{Z} \mathcal{G}(\mathcal{F})(G / H, G / K) \otimes M
\end{aligned}
$$


for any trivial $\mathbf{Z} G$-module $M$. This gives

$$
\begin{aligned}
W_{n}(T, \mathcal{F})^{H} \cong & \bigoplus C\left(G / H, G / H_{0}\right) \\
& \otimes\left[C\left(G / H_{0}, G / H_{1}\right) \otimes \cdots \otimes C\left(G / H_{n-1}, G / H_{n}\right)\right] \otimes T\left(G / H_{n}\right),
\end{aligned}
$$

with the notation of 2.4. Under this identification, define $\epsilon: W_{0}(T, \mathcal{F})^{H} \rightarrow T(G / H)$ by taking a summand $f[] t$ to $f^{*}(t)$. A contracting chain homotopy is then given by

$$
s_{n}\left(f\left[f_{1}, \ldots, f_{n}\right] t\right)=\left(1_{H}\left[f, f_{1}, \ldots, f_{n}\right] t\right)
$$

where $1_{H}$ is the identity morphism $G / H \rightarrow G / H$. It is now formal that $d s-s d=$ 1.

REMARKS 2.6. (i) Note that $W_{*}(T, \mathcal{F})^{H}$ is not acyclic as a $\mathbf{Z} N(H)$-module; the contracting homotopy does not respect the $N(H)$-action.

(ii) If $\mathcal{F}=\{1\}$ and $T=\mathbf{Z}$, then $W_{*}(T, \mathcal{F})$ is a free $\mathbf{Z} G$-resolution of the trivial $G$-module $\mathbf{Z}$, and is, in fact, isomorphic with the usual Bar resolution.

(iii) $\mathbf{Z} G / H$ is not, in general, a projective $\mathbf{Z} G$-module. It is therefore inappropriate to view $W_{*}(T, \mathcal{F})$ as a resolution in the category of $\mathbf{Z} G$-modules.

Regard $W_{*}(T, \mathcal{F})$ as an $\mathcal{F}$-Hecke functor as in 2.3 ; we set $W_{*}(T, \mathcal{F})(G / H)=$ $W_{*}(T, \mathcal{F})^{H} \cong \operatorname{Hom}_{\mathbf{Z}}\left(\mathbf{Z} G / H, W_{*}(T, \mathcal{F})\right.$ ). (Note that this isomorphism demonstrates the action of $\mathbf{Z G}(\mathcal{F})$ as the obvious left one.)

Proposition 2.7. Let $H \in \mathcal{F}$. Then $\mathbf{Z} G / H$ is a projective $\mathcal{F}$-Hecke functor.

ProOF. Denote the category of $\mathcal{F}$-Hecke functors by $\mathcal{M}(\mathcal{F})$. We now claim that

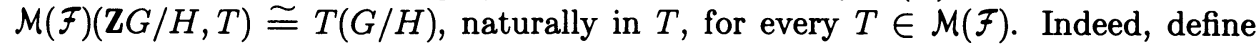
$\phi: \mathcal{M}(\mathcal{F})(\mathbf{Z} G / H, T) \rightarrow T(G / H)$ by taking $f$ to $f(G / H)(e H)$ and $\psi: T(G / H) \rightarrow$ $\mathcal{M}(\mathcal{F})(\mathbf{Z} G / H, T)$ by taking $\psi(t)(G / K)$ to be the composite

$$
(\mathbf{Z} G / H)^{K} \stackrel{\circ}{=} \operatorname{Hom}_{\mathbf{Z} G}(\mathbf{Z} G / K, \mathbf{Z} G / H) \stackrel{\epsilon}{\rightarrow} T(G / K),
$$

where $\epsilon(r)=T(r)(t)$. Thus $\psi(t)(G / K)(x)=T(\sigma(x))(t)$. That $\psi(t)$ is indeed a morphism in $\mathcal{M}(\mathcal{F})$ follows by the naturality of the definition of $\psi$, and that $\phi \psi=$ 1 is clear. That $\psi \phi$ is the identity is a consequence of the following. Given any morphism $f: \mathbf{Z} G / H \rightarrow T$ in $\mathcal{M}(\mathcal{F})$ and $x \in(\mathbf{Z} G / H)^{K}$ with $K \in \mathcal{F}$, the diagram

$$
\begin{array}{cc}
(\mathbf{Z} G / H)^{H} \stackrel{f(G / H)}{\rightarrow} & T(G / H) \\
\sigma_{(x)^{*}} \downarrow & \downarrow T(\sigma(x)) \\
(\mathbf{Z} G / H)^{K} \stackrel{f(G / K)}{\rightarrow} T(G / K)
\end{array}
$$

must commute, where $\sigma(x)^{*}$ is induced by $\sigma(x): \mathbf{Z} G / K \rightarrow \mathbf{Z} G / H ; e K \mapsto x$. Chasing $e H$ around the diagram gives

$$
f(G / K)\left(\sigma(x)^{*} e H\right)=f(G / K)(x)=T(\sigma(x))(f(G / H)(e H)),
$$

showing that $f(G / K)(x)$ is completely specified by $f(G / H)(e H)$ via application of $T(\sigma(x))$. This shows that $\psi \phi=1$ as claimed.

For projectivity of $\mathbf{Z} G / H$ as an object in $\mathcal{M}(\mathcal{F})$, we now observe that one may complete any diagram of the form

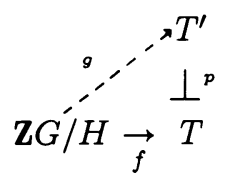


in $\mathcal{M}(\mathcal{F})$ by setting $g=\psi(t)$ where $t \in T^{\prime}(G / H)$ is any element in $p^{-1}(f(G / H)(e H))$.

REMARKS 2.8. If $H \notin \mathcal{F}$, then the assertion is false; let $\mathcal{F}=\{1\}$ and $H=G$. Then $\mathbf{Z} G / G=\mathbf{Z}$ is not a projective $\mathbf{Z} G$-module. Hence it is not projective in $\mathcal{M}(\mathcal{F})$, as one notes that if $\mathcal{F}=\{1\}, \mathcal{M}(\mathcal{F})$ is equivalent to the category of $\mathbf{Z} G$-modules. $\mathbf{Z}$ is, however, a projective $\mathcal{M}(\{1, g\})$-module by the proposition.

If each $T(G / H)$ is a projective $\mathbf{Z}$-module, then it follows that $W_{*}(T, \mathcal{F})$ is a projective resolution of $T$ in the category $\mathcal{M}(\mathcal{F})$. (One should not expect the Bar resolution to yield projective modules in general - even in the case $G$ trivial.) As far as the applications are concerned, we may take each $T(G / H)$ to be projective. In general, there are "enough" projectives; if $T \in \mathcal{M}(\mathcal{F})$, and if $F(G / H)$ is the free abelian group on the elements of $T(G / H)$, then the map

$$
\epsilon: \bigoplus_{H \in \mathcal{F}} \mathbf{Z} G / H \otimes F(G / H) \rightarrow T
$$

of $\mathcal{F}$-Mackey functors, defined just as in the proof of Proposition 2.5, is an epimorphism in $\mathcal{M}(\mathcal{F})$.

Now let $M_{*}$ be any d.g. $\mathcal{F}$-Hecke functor, and let $T \in \mathcal{M}(\mathcal{F})$.

DEFINITION 2.9. The cohomology of $M_{*}$ with $T$-coefficients, $H^{*}\left(M_{*} ; T\right)$, is defined to be $H^{*}\left(\mathcal{M}(\mathcal{F})\left(M_{*}, T\right)\right.$ ), (where, as usual, $\mathcal{M}(\mathcal{F})(-,-)$ denotes Hom in the category $\mathcal{M}(\mathcal{F})$ ).

EXAMPLES 2.10. (i) Let $X$ be any $G$-CW complex with cells of type $G / H \times D^{n}$ with $H \in \mathcal{F}$. Then the cellular chain complex, $C_{*}(X)$ is a d.g. $\mathcal{F}$-Hecke functor if we define $C_{*}(X)(G / H)=C_{*}(X)^{H}\left(\neq C_{*}\left(X^{H}\right)\right)$. Since $C_{*}(X)$ is a sum of $G$-modules $\mathbf{Z} G / H$ with $H \in \mathcal{F}$, it is a projective $\mathcal{F}$-Hecke functor by Proposition 2.7. We shall see in $\S 3$ that $H^{*}\left(C_{*}(X) ; T\right)$ is just Bredon cohomology, $H_{G}^{*}(X ; T)$, as in [B1], provided $\mathcal{F}$ has the property that $H \in \mathcal{F}, K \subset H$ implies $K \in \mathcal{F}$. (This condition also allows one to think of $T$ as a coefficient system defined on all orbit-types, so that $H_{G}^{*}(-; T)$ is meaningful.)

(ii) If $M_{*}=W_{*}(T, \mathcal{F})$ with $\mathcal{F}=\{1\}$ and $T=\hat{\mathbf{Z}}$, it follows that $H^{*}\left(M_{*} ; \hat{R}\right) \cong$ $H^{*}(G ; R)$ for all $\mathbf{Z} G$-modules $R$.

(iii) In general, define $H^{*}\left((T, \mathcal{F}) ; T^{\prime}\right)$ to be $H^{*}\left(W_{*}(T, \mathcal{F}) ; T^{\prime}\right)$. This generalizes the notion of $H^{*}(G ; R)$ to larger families of subgroups, and is independent of the choice of resolution $W_{*}(T, \mathcal{F})$ if each $T(G / H)$ is projective.

3. Applications. Let $\mathcal{F}$ be a family of subgroups with the property that, if $H \in \mathcal{F}$ and $g K g^{-1} \subset H$ for some $g \in G$, then $K \in \mathcal{F}$.

By a universal $G$-space $E \mathcal{F}$, we shall mean a $G$-space with $E \mathcal{F}^{H}$ contractible for each $H \in \mathcal{F}$, and $E \mathcal{F}^{H}=\emptyset$ otherwise. (For example, $E\{1\}=E G$, the standard universal $G$-space.) These spaces were first constructed by Palais, while Elmendorf has an elegant construction in terms of the geometric two-sided Bar construction in [E1]. As a consequence of these constructions, we may assume that $E$ is a $G-C W$ complex with cells of type $G / H \times D^{n}$ and $H \in \mathcal{F}$.

If $T$ is in $\mathcal{M}(\mathcal{F})$, we consider the problem of computing $H_{G}^{*}(E \mp ; T)$, the Bredon cohomology of $E \mathcal{F}$. (As a special case, $H_{G}^{*}(E G ; \hat{R}) \cong H^{*}(G ; R)$.)

As promised in $\S 2$, we have the following result.

LEMMA 3.1. Let $X$ be any $G$-CW complex with cells of type $G / H \times D^{n}$ with $H \in \mathcal{F}$. Then $H^{*}\left(C_{*}(X) ; T\right) \cong H_{G}^{*}(X ; T)$, Bredon cohomology of $X$ with coefficients in the coefficient system $T$, where we take $T(G / K)=0$ if $K \notin \mathcal{F}$. 
PROOF. It suffices to construct a natural transformation

$$
\psi: H^{*}\left(C_{*}(X) ; T\right) \rightarrow H_{G}^{*}(X ; T)
$$

which restricts to an isomorphism where $X=G / H$ and $H \in \mathcal{F}$, and to verify that $H^{*}\left(C_{*}(X) ; T\right)$ is indeed a generalized equivariant cohomology theory in the sense of Bredon.

The latter claim is clear as a consequence of the projectivity of $C_{*}(X)$. For the former, recall from [B1] that $H_{G}^{*}(X ; T)$ is the cohomology of $\operatorname{Hom}\left(\underline{C}_{*}(X), T\right)$ in the category of coefficient systems, where $\underline{C}_{*}(X)(G / H)=C_{*}\left(X^{H}\right)$ for all $H \subset G$. Since $X^{H}=\emptyset$ for $H \notin \mathcal{F}$, it follows that $\underline{C}_{*}(X)(G / H)=0$ for such $H$. Now define $\psi: \mathcal{M}(\mathcal{F})\left(C_{*}(X), T\right) \rightarrow \operatorname{Hom}\left(\underline{C}_{*}(X), T\right)$ by

$$
f(G / H) \mapsto \begin{cases}f(G / H) \mid C_{*}\left(X^{H}\right) & \text { if } H \in \mathcal{F} ; \\ 0 & \text { if } H \notin \mathcal{F} .\end{cases}
$$

Then $\psi$ induces the desired transformation after passage to cohomology. Further, if $H \in \mathcal{F}$, then $\mathcal{M}(\mathcal{F})\left(C_{*}(G / H), T\right) \cong \mathcal{M}(\mathcal{F})(\mathbf{Z} G / H, T) \cong T(G / H)$, and under this isomorphism, $\psi$ becomes the identity.

PROPOSITION 3.2. There exists an isomorphism, natural in $T$,

$$
\phi: H_{G}^{*}(E \mp ; T) \cong H^{*}((\hat{\mathbf{Z}}, \mathcal{\Im}) ; T) \text {. }
$$

Proof. By the lemmas,

$$
H_{G}^{*}(E \mathcal{F} ; T) \cong H^{*}\left(C_{*}(E \mathcal{F}) ; T\right) \text {, and } C_{*}(E \mathcal{F})^{H} \cong C_{*}(E \mathcal{F} / H)
$$

for all $H \in \mathcal{F}$. Further, since $E \mathcal{F}^{K}$ is contractible for each $K \subset H$, it follows that $E \mathcal{F}$ is $H$-equivariantly contractible, whence $E \mathcal{F} / H$ is contractible. Thus $C_{*}(E \mp)$ is a projective resolution of $\hat{\mathbf{Z}}$ in $\mathcal{M}(\mathcal{F})$, and the result now follows by the lemma.

REMARKS 3.3. Proposition 3.2 permits explicit computation of $H_{G}^{*}(E \mathcal{F} ; T)$ since one can write down the appropriate Bar resolution $W_{*}(T, \mathcal{F})$. It also follows that $H_{G}^{*}(E \mathcal{F} ; T)$ is an algebraic invariant of $G, \mathcal{F}$ and $T$. Of course, when $T$ is replaced by $\hat{R}$ and $\mp$ by $\{1\}$, we obtain the expected isomorphism $H_{G}^{*}(E G ; \hat{R}) \cong$ $H^{*}(G ; R)$.

For the next application, we permit $₹$ to denote any family of subgroups closed under conjugation.

Proposition 3.4. Let $X$ be a $G$-CW complex of dimension $n$ such that $X / H$ is $\mathbf{Z}$-acyclic for each $H \in \mathcal{F}$, and such that $X$ has all orbits of the form $G / H$ with $H \in \mathcal{F}$. Then $H^{p}((\hat{\mathbf{Z}}, \mathcal{F}) ; T)=0$ for all $p>n$.

PROOF. $C_{*}(X)$ is, under the hypothesis, a projective resolution of $\hat{\mathbf{Z}}$ in $\mathcal{M}(\mathcal{F})$, where $H^{p}\left(C_{*}(X) ; T\right) \cong H^{p}((\hat{\mathbf{Z}}, \mathcal{F}) ; T)$ for all $p$. Since the former group vanishes for $p>n$, the result follows.

COROLlARY 3.5. Let $G$ act on a space $X$ such that

(i) $X$ inherits the structure of a contractible G-CW complex;

(ii) $\operatorname{dim} X=n$;

(iii) $X$ has orbit-types $G / H$ with $H \in \mathcal{F}$.

Then $H^{p}((\hat{\mathbf{Z}}, \mathcal{F}) ; T)=0$ for all $p>n$.

Proof. By $[\mathbf{B 2}, 5.4], C_{*}(X / H)$ is Z-acyclic for each $H \subset G$. The result now follows by 3.4 . 
Corollary 3.6. Let $G \in \mathcal{F}$. Then

for all $T$.

$$
H^{p}((\hat{\mathbf{Z}}, \mathcal{F}) ; T) \cong \begin{cases}0 & \text { if } p>0, \\ T(G / G) & \text { if } p=0\end{cases}
$$

Proof. We apply Corollary 3.5 with $X=$ point, obtaining $H^{p}((\hat{\mathbf{Z}}, \mathcal{\xi}) ; T)=0$ for $p>0$. The case $p=0$ is then obtained by writing out $C *$ (point) and observing that $\mathcal{M}(\mathcal{F})(\hat{\mathbf{Z}}, T) \cong T(G / G)$, as in the proof of 2.7 .

Corollary 3.5 gives the lowest possible dimension of a finite contractible $\mathrm{CW}$ complex $X$ with a suitable $G$-action. This problem has been examined by Oliver and Assadi (see [A1]), and they obtain necessary and sufficient conditions on the subgroups of $G$ for such actions to exist.

\section{BIBLIOGRAPHY}

[A1] A. Assadi, Thesis, Princeton Univ., Princeton, N. J., 1979.

[B1] G. E. Bredon, Equivariant cohomology theories, Lecture Notes in Math., vol. 34, SpringerVerlag, Berlin and New York, 1967.

[B2] - Introduction to compact transformation groups, Academic Press, New York, 1972.

[D1] T. tom Dieck, Transformation groups and representation theory, Lecture Notes in Math., vol. 766, Springer-Verlag, Berlin and New York, 1979.

[E1] A. Elmendorf, Thesis, Univ. of Chicago, Chicago, Ill., 1979.

[G1] J. A. Green, Axiomatic representation theory for finite groups, J. Pure Appl. Math. 1 (1971), 41-47.

[RS] K. Roggenkamp and L. Scott, Hecke actions on Picard groups, Preprint, Univ. of Virginia, Charlottesville, 1981.

[W1] S. Waner, G-CW(V) complexes, Preprint, Univ. of Virginia, Charlottesville, 1980.

Department of Mathematics, UNiversity of Virginia, Charlottesville, VIRGINIA 22903 